TRANSACTIONS OF THE

AMERICAN MATHEMATICAL SOCIETY

Volume 350, Number 10, October 1998, Pages 4041-4051

S 0002-9947(98)02128-X

\title{
TEST IDEALS IN QUOTIENTS OF F-FINITE REGULAR LOCAL RINGS
}

\author{
JANET COWDEN VASSILEV
}

\begin{abstract}
Let $S$ be an $F$-finite regular local ring and $I$ an ideal contained in $S$. Let $R=S / I$. Fedder proved that $R$ is $F$-pure if and only if $\left(I^{[p]}: I\right)$ $\nsubseteq \mathfrak{m}^{[p]}$. We have noted a new proof for his criterion, along with showing that $\left(I^{[q]}: I\right) \subseteq\left(\tau^{[q]}: \tau\right)$, where $\tau$ is the pullback of the test ideal for $R$. Combining the the $F$-purity criterion and the above result we see that if $R=S / I$ is $F$ pure then $R / \tau$ is also $F$-pure. In fact, we can form a filtration of $R, I \subseteq \tau=$ $\tau_{0} \subseteq \tau_{1} \subseteq \ldots \subseteq \tau_{i} \subseteq \ldots$ that stabilizes such that each $R / \tau_{i}$ is $F$-pure and its test ideal is $\tau_{i+1}$. To find examples of these filtrations we have made explicit calculations of test ideals in the following setting: Let $R=T / I$, where $T$ is either a polynomial or a power series ring and $I=P_{1} \cap \ldots \cap P_{n}$ is generated by monomials and the $R / P_{i}$ are regular. Set $J=\Sigma\left(P_{1} \cap \ldots \cap \hat{P}_{i} \cap \ldots \cap P_{n}\right)$. Then $J=\tau=\tau_{\text {par }}$.
\end{abstract}

This paper concerns the study of the test ideal in $F$-finite quotients of regular local rings. Test elements play a key role in tight closure theory. Once known, they make computing tight closures of ideals and modules easier. In fact, in an excellent Gorenstein local ring with an isolated singularity, $R / \tau \cong \operatorname{Hom}\left(I^{*} / I, E\right)$ where $I$ is generated by a system of parameters that are test elements and $E$ is the injective hull of $R$ (see [Hu1] and [S1]). We also know for parameter ideals $I$ that $I: \tau=I^{*}$. Thus knowing $\tau$ is basically equivalent to knowing the tight closure of a system of parameters which is contained in the test ideal. A recent paper of Huneke and Smith [HS] links tight closure to Kodaira vanishing for graded rings $R$ with characteristic either 0 or $p$ where $p \gg 0$. Recall that the $a$-invariant for a graded ring $R$, denoted $a$, is equal to $-\min \left\{i \mid\left[\omega_{R}\right]_{i} \neq 0\right\}$, where $\omega_{R}$ is the canonical module for $R$. If $R$ is Gorenstein, then $\omega_{R}=R(a)$. Huneke and Smith prove that the test ideal is exactly the ideal generated by elements of degree greater than the $a$-invariant of $R$ if and only if a strong Kodaira vanishing holds on $R$. A recent paper of Hara [Ha] confirms that this strong Kodaira vanishing holds in finitely generated algebras over a field of characteristic zero.

In this paper we study test ideals of $F$-finite rings which are reduced quotients of a regular local ring. Reduced quotients of $F$-finite regular local rings have been studied by both Fedder [Fe] and Glassbrenner [Gl]. Fedder's work concerns Fpurity aspects of these rings, and Glassbrenner's results use Fedder's techniques to examine strong $F$-regularity. The object that plays a key role in their work is

Received by the editors November 4, 1996.

1991 Mathematics Subject Classification. Primary 13A35.

Key words and phrases. Tight closure, test element, $F$-finite, $F$-pure.

I would like to express my appreciation to Purdue University for hosting me during the time that I completed these results. I also thank Craig Huneke for many helpful conversations. 
$\left(I^{[q]}: I\right)$, where $R=S / I, S$ a regular local ring and $I$ is an ideal in $S$. In section 2 we introduce Fedder's key result [Fe], which states that $R$ is $F$-pure if and only if $\left(I^{[q]}: I\right) \nsubseteq \mathfrak{m}^{[q]}$. We also develop techniques to simplify the proof for his main result. In section 3 we go on to show that $\left(I^{[q]}: I\right) \subseteq\left(\tau^{[q]}: \tau\right)$, and we combine this result with Fedder's $F$-purity criterion to conclude that the ring $R / \tau$ is also $F$-pure. We note that by successively applying our result and Fedder's $F$-purity criterion we find a unique filtration of the ring $R, I \subseteq \tau_{0} \subseteq \tau_{1} \subseteq \ldots \subseteq \tau_{i} \ldots$ where $\tau_{i} / \tau_{i-1}$ is the test ideal of the ring $R / \tau_{i-1}$ and the $R / \tau_{i}$ are all $F$-pure. In order to give some examples of this filtration we compute the test ideal for regular rings modulo square free monomial ideals. In section 4 over $F$-pure rings we examine what power of the tight closure of an ideal is contained in the ideal itself.

\section{Preliminaries}

Let $R$ be a commutative Noetherian ring of prime characteristic $p>0$ and $I$ be an ideal of $R$ with generators $x_{1}, \ldots, x_{n}$. Denote powers of $p$ by $q$. Define $R^{o}$ to be the complement of the union of minimal primes.

Definition 1.1. We say $x \in R$ is in the tight closure of $I$ if there exists a $c \in R^{o}$ such that $c x^{q} \in I^{[q]}$ for all large $q$, where $I^{[q]}=\left(x_{1}^{q}, \ldots, x_{n}^{q}\right)$. Denote the tight closure of $I$ by $I^{*}$.

As one might expect, the Frobenius map plays an active role in tight closure theory, and we pay tribute to it in most tight closure notions. Recall that the Frobenius map, $F: R \rightarrow R$ is defined by the following action on $R: F(r)=r^{p}$. We denote $R$ viewed as an $R$-module via the Frobenius map as $F(R)$. If $F(R)$ is a finite $R$-module then we call $R F$-finite. A monomorphism $f: R \rightarrow S$ is pure if $f \otimes 1_{M}: R \otimes M \rightarrow S \otimes M$ is injective for all $R$-modules $M$. If $R \rightarrow F(R)$ is pure, then we say $R$ is $F$-pure. Fedder and Watanabe [FW] define $R$ to be $F$-injective if $H_{\mathfrak{m}}^{n}(R) \rightarrow H_{\mathfrak{m}}^{n}(F(R))$ is injective for all $n$. They note that when $R$ is Cohen-Macaulay, $R$ is $F$-injective if $x^{p} \in I^{[p]}$ implies $x \in I$ for parameter ideals $I$.

In the definitions of tight closure of ideals and modules above we observe that the element $c$ must be chosen in $R^{o}$. If $c \in \bigcap_{J \subseteq R}\left(J: J^{*}\right)$, then we call $c$ a test element.

Definition 1.2. The ideal generated by the test elements is called the test ideal and we denote it by $\tau$.

We can also define the parameter test ideal to be the ideal generated by all elements $c$ that multiply all $J^{*}$ into $J$ where $J$ is a parameter ideal. The parameter test ideal is by denoted $\tau_{\text {par }}$.

\section{F-FINITENESS "CRITERIA"}

$(S, \mathfrak{m})$ will always be an $F$-finite regular local ring. Set $R=S / I$. It is important to note that $F^{e}(S)$ is a free $S$-module [Fe]. Fedder used the freeness of $F^{e}(S)$ to prove his $F$-purity criterion for quotients of regular local rings. The author exploits this freeness in a different way to give a new proof.

Lemma 2.1. Let $S$ be a Noetherian ring and $M$ a free $S$-module. Then $\bigcap_{i \in I} I_{i} M=$ $\left(\bigcap_{i \in I} I_{i}\right) M$, where $\left\{I_{i}\right\}_{i \in I}$ are ideals in $S$. In particular, if $S$ is an $F$-finite regular 
local ring and $\left\{I_{i}\right\}$ a collection of ideals in $S$, then

$$
\left(\bigcap I_{i}\right)^{[q]}=\left(\bigcap I_{i}\right) F^{e}(S) \cap S=\left(\bigcap I_{i} F^{e}(S)\right) \cap S=\left(\bigcap I_{i}^{[q]}\right),
$$

where $q=p^{e}$.

Proof. Let $\left\{x_{j}\right\}_{j \in J}$ be a basis for $M$ over $S$. Then $M=\bigoplus_{j \in J} S x_{j}$. We claim that for any ideal $\mathfrak{A} \subseteq S, \mathfrak{A} M=\bigoplus_{j \in J} \mathfrak{A} x_{j}$. Thus we can consider $\left(\bigcap_{i \in I} I_{i}\right) M=$ $\bigoplus_{j \in J}\left(\bigcap_{i \in I} I_{i}\right) x_{j}$. Trivially we can see that $\left(\bigcap_{i \in I} I_{i}\right) M \subseteq \bigcap_{i \in I}\left(I_{i} M\right)$. To see the other inclusion we notice that if $x \in \bigcap_{i \in I}\left(I_{i} M\right)$, then $x \in I_{i} M$ for all $i \in I$. Now $x=\sum_{j \in J} a_{j} x_{j}$, where $a_{j} \in I_{i}$ for all $i \in I$ and $j \in J$, which implies $a_{j} \in \bigcap_{i \in I} I_{i}$. Thus $x \in\left(\bigcap_{i \in I} I_{i}\right) M$.

We note another lemma before we proceed with the new proof for the above mentioned criterion.

Lemma 2.2. Let $\left\{J_{n}\right\}$ be a collection of ideals in $S$ such that $\bigcap J_{n}=I$. Then $\bigcap\left(J_{n}^{[q]}: I\right)=\left(I^{[q]}: I\right)$.

Proof. Lemma 2.1 implies $\bigcap J_{n}^{[q]}=I^{[q]}$. Since $I^{[q]} \subseteq J_{n}^{[q]}$ for all $n \in \mathbb{N}$, it follows that $\left(I^{[q]}: I\right) \subseteq \bigcap\left(J_{n}^{[q]}: I\right)$. To see the opposite containment we need only prove that $\bigcap\left(J_{n}^{[q]}: I\right) \subseteq\left(I^{[q]}: I\right)$. Let $x \in \bigcap\left(J_{n}^{[q]}: I\right)$. Then $I x \subseteq \bigcap_{n \in \mathbb{N}} J_{n}^{[q]}=I^{[q]}$. In other words, $x \in\left(I^{[q]}: I\right)$.

Besides the freeness of $F^{e}(S)$ over $S$, we wish to use the fact that $R=S / I$ is approximately Gorenstein, i.e. there exist irreducible ideals $\left\{q_{n}\right\}$ cofinal with $\mathfrak{m}$ such that $R / q_{n}$ is a 0 -dimensional Gorenstein ring for all $n \in \mathbb{N}$. To see that $R=S / I$ is approximately Gorenstein, recall that $R=S / I$ was a reduced ring and two theorems from Hochster [Ho]:

Theorem 2.3. [Ho, 1.7] Let $R$ be a locally excellent Noetherian ring. If $R$ is reduced and $S$ is any extension algebra of $R$ where $R$ is cyclically pure in $S$, then $R$ is pure.

Theorem 2.4. [Ho, 2.3] The following are equivalent:

1) $R$ is approximately Gorenstein.

2) If $S$ is an extension algebra of $R$ with $R$ cyclically pure in $S$, then $R$ is pure.

Combining 2.3 and 2.4 we see that $R$ is approximately Gorenstein. Hochster also proves that $R$ is approximately Gorenstein if and only if the $\mathfrak{m}$-adic completion $\hat{R}$ is approximately Gorenstein [Ho, 1.6].

Since both completion and the application of the Frobenius are faithfully flat over regular local rings [Ku1], we can reduce to the case where $\mathrm{R}$ is complete. Now we give a new proof of Fedder's $F$-purity criterion:

Theorem 2.5. [Fe, Proposition 1.7] Let $R=S / I$, where $S$ is an F-finite regular local ring. Then $R$ is F-pure if and only if $\left(I^{[p]}: I\right) \nsubseteq \mathfrak{m}^{[p]}$.

Proof. $(\Rightarrow)$ Since completion and application of the Frobenius are both faithfully flat over regular local rings as noted above, we may reduce to the case where both $R$ and $S$ are complete. Since $R$ is a complete local ring we see that it is approximately Gorenstein by [Ho]. Thus we have irreducible ideals $q_{n}$ containing $I$ with $R / q_{n} 0$ dimensional Gorenstein. Note that $\bigcap_{n \geq 1} q_{n} \subseteq \bigcap_{N \geq 1}\left(\mathfrak{m}^{N}+I\right)=I$. However, $I \subseteq q_{n}$ for all $n \in \mathbb{N}$ implies $\bigcap q_{n}=I$. 
Since $R / q_{n}$ is a 0 -dimensional Gorenstein ring,

$$
\operatorname{Soc}\left(R / q_{n}\right) \cong R / \mathfrak{m},
$$

i.e. $\operatorname{Soc}\left(R / q_{n}\right)=\left(q_{n}: \mathfrak{m}\right)$ can be generated by one element, say $x_{n} \notin q_{n}$. We know from $\left[\mathrm{BH}\right.$, exercise 3.2.15] that $\left(q_{n}:\left(q_{n}: \mathfrak{m}\right)\right)=\mathfrak{m}$. Thus $\left(q_{n}: x_{n}\right)=\mathfrak{m}$. Since the Frobenius is faithfully flat, $\left(q_{n}^{[p]}: x_{n}^{p}\right)=\mathfrak{m}^{[p]}$.

By Lemma 2.2 we know that $\bigcap\left(q_{n}^{[p]}: I\right)=\left(I^{[p]}: I\right)$. Since $\left(q_{n}^{[p]}: I\right)$ are a descending chain of ideals in $S$, if $\left(I^{[p]}: I\right) \subseteq \mathfrak{m}^{[p]}$, then for some large $n$,

$$
\left(q_{n}^{[p]}: I\right) \subseteq \mathfrak{m}^{[p]}=\left(q_{n}^{[p]}: x_{n}^{p}\right) .
$$

Hence $\left(q_{n}^{[p]}:\left(q_{n}^{[p]}: x_{n}^{p}\right)\right) \subseteq\left(q_{n}^{[p]}:\left(q_{n}^{[p]}: I\right)\right)$. But $x_{n}^{p} \in\left(q_{n}^{[p]}: \mathfrak{m}^{[p]}\right)=\left(q_{n}: \mathfrak{m}\right)^{[p]}$. Thus $x_{n}^{p} \in\left(q_{n}^{[p]}:\left(q_{n}^{[p]}: I\right)\right)$. To see that $\left(q_{n}^{[p]}:\left(q_{n}^{[p]}: I\right)\right)=q_{n}^{[p]}+I$, we need only show that

$$
\left(q_{n}^{[p]}: I\right)=\left(q_{n}^{[p]}: q_{n}^{[p]}+I\right) .
$$

First note that $q_{n}^{[p]}+I \subseteq\left(q_{n}^{[p]}:\left(q_{n}^{[p]}: I\right)\right)$. Thus

$$
\left(q_{n}^{[p]}: I\right)=\left(q_{n}^{[p]}:\left(q_{n}^{[p]}:\left(q_{n}^{[p]}: I\right)\right)\right) \subseteq\left(q_{n}^{[p]}: q_{n}^{[p]}+I\right) .
$$

However, $I \subseteq q_{n}^{[p]}+I$ implies that

$$
\left(q_{n}^{[p]}: q_{n}^{[p]}+I\right) \subseteq\left(q_{n}^{[p]}: I\right) .
$$

Thus $x_{n}^{p} \in q_{n}^{[p]}+I$. Since $x_{n}$ was chosen not in $q_{n}$, and $x_{n}^{p} \in q_{n}^{[p]}, R$ is not $F$-pure.

$(\Leftarrow)$ Suppose $\left(I^{[p]}: I\right) \nsubseteq \mathfrak{m}^{[p]}$. Let $J$ be an ideal of $S$ containing $I$. If $x^{p} \in J^{[p]}+I$, then $\left(I^{[p]}: I\right) x^{p} \subseteq J^{[p]}$. Thus $\left(I^{[p]}: I\right) \subseteq(J: x)^{[p]}$ and $(J: x)^{[p]}$ is not contained in $\mathfrak{m}^{[p]}$ by assumption. Thus $x \in J$, and thus $R$ is $F$-pure.

Fedder has shown the above criterion for $q=p$. It is fairly easy to show when $q=p^{e}($ where $e \neq 1)$ that $\left(I^{[q]}: I\right) \nsubseteq \mathfrak{m}^{[q]}$ is equivalent to $\left(I^{[p]}: I\right) \nsubseteq \mathfrak{m}^{[p]}$. Thus we can check $F$-purity on a quotient of an $F$-finite regular local ring for any power of $p$.

Theorem 2.6. Let $R=S / I$ where $S$ is an $F$-finite regular local ring. Then for $q=p^{e}$ where $e \neq 1$ we have $\left(I^{[q]}: I\right) \nsubseteq \mathfrak{m}^{[q]}$ if and only if $\left(I^{[p]}: I\right) \nsubseteq \mathfrak{m}^{[p]}$.

Proof. $(\Rightarrow)$ Suppose $\left(I^{[p]}: I\right) \subseteq \mathfrak{m}^{[p]}$. Apply the $(e-1)$ st power of the Frobenius to $\operatorname{get}\left(I^{[p]}: I\right)^{[q / p]}=\left(I^{[q]}: I^{[q / p]}\right) \subseteq \mathfrak{m}^{[q]}$. But $\left(I^{[q]}: I\right) \subseteq\left(I^{[q]}: I^{[q / p]}\right) \subseteq \mathfrak{m}^{[q]}$, which is a contradiction.

$(\Leftarrow)$ Suppose there exists a $q$ such that $\left(I^{[q]}: I\right) \subseteq \mathfrak{m}^{[q]}$. Since $S$ is regular, $\mathfrak{m}=\left(x_{1}, \ldots, x_{d}\right)$, where $x_{1}, \ldots, x_{d}$ is a system of parameters. The socle of $S / \mathfrak{m}^{[p]}$ is

$$
\left(\mathfrak{m}^{[p]}:_{\mathfrak{m}[p]} \mathfrak{m}\right)=\left(\mathfrak{m}^{[p]},\left(x_{1} \ldots x_{d}\right)^{p-1}\right) / \mathfrak{m}^{[p]} .
$$

If $\left(I^{[p]}: I\right) \nsubseteq \mathfrak{m}^{[p]}$, then there exists $x \in\left(I^{[p]}: I\right)$ such that $x$ generates the socle of $S / \mathfrak{m}^{[p]}$. We can conclude that $x=\left(x_{1} \ldots x_{d}\right)^{p-1}+a_{1} x_{1}^{p}+\ldots a_{d} x_{d}^{p}$. Note that $\prod_{i=0}^{n-1} x^{p^{i}} \in \prod_{i=0}^{n-1}\left(I^{[p]}: I\right)^{\left[p^{i}\right]}=\prod_{i=0}^{n-1}\left(I^{\left[p^{i+1}\right]}: I^{\left[p^{i}\right]}\right) \subseteq\left(I^{\left[p^{n}\right]}: I\right)$. By an easy computation we see that $\prod_{i=0}^{n-1} x^{p^{i}}=x^{\frac{p^{n}-1}{p-1}}=\left(x_{1} \ldots x_{d}\right)^{p^{n}-1}+b_{1} x_{1}^{p^{n}}+\ldots+b_{d} x_{d}^{p^{n}}$ for some $b_{i} \in R$. But this is in the socle of $S / \mathfrak{m}^{\left[p^{n}\right]}$. Thus $\left(I^{\left[p^{n}\right]}: I\right) \nsubseteq \nsubseteq \mathfrak{m}^{\left[p^{n}\right]}$. 


\section{TEST IDEAls in $F$-Finite REDUCED RINGS}

Using some of the same techniques from the previous section we can show the following:

Theorem 3.1. Let $S$ be an F-finite regular local ring, $R=S / I$. If $f: S \rightarrow R$ is the canonical surjection, then $\left(I^{[p]}: I\right) \subseteq\left(f^{-1}\left(\tau_{R}\right)^{[p]}: f^{-1}\left(\tau_{R}\right)\right)$.

Proof. Denote $J / I \subseteq R$ by $\bar{J}$. Notice that for all $\bar{J} \subseteq R, \tau_{R}\left(\bar{J}^{*}\right)^{[p]} \subseteq \bar{J}^{[p]}$. For elements $c \in \tau_{R}$ and $x \in \bar{J}^{*}$ we have that $c x^{p} \in \bar{J}^{[p]}$. In other words, after pulling elements and ideals back to $S$ we have $\left(c+i_{i}\right)\left(x^{p}+i_{2}\right) \in J^{[p]}+I$ for all $i_{1}$ and $i_{2}$ in $I$. Thus

$$
f^{-1}\left(\tau_{R}\right)\left(f^{-1}\left(\bar{J}^{*}\right)\right)^{[p]} \subseteq J^{[p]}+I .
$$

Let $w \in\left(I^{[p]}: I\right)$, so that $w f^{-1}\left(\tau_{R}\right)\left(f^{-1}\left(\bar{J}^{*}\right)\right)^{[p]} \subseteq J^{[p]}+I^{[p]} \subseteq J^{[p]}$ in $S$. Thus

$$
w f^{-1}\left(\tau_{R}\right) \subseteq\left(J^{[p]}:_{S}\left(f^{-1}\left(\bar{J}^{*}\right)\right)^{[p]}\right)=\left(J:_{S} f^{-1}\left(\bar{J}^{*}\right)\right)^{[p]} .
$$

So $w f^{-1}\left(\tau_{R}\right) \subseteq \bigcap_{I \subseteq J}\left(J:_{S} f^{-1}\left(J^{*}\right)\right)^{[p]}$. Using Lemma 2.1, we see that

$$
\bigcap_{I \subseteq J}\left(J: S f^{-1}\left(\bar{J}^{*}\right)\right)^{[p]}=\left(\bigcap_{I \subseteq J}\left(J:_{S} f^{-1}\left(\bar{J}^{*}\right)\right)\right)^{[p]} .
$$

As long as $\bigcap_{I \subseteq J}\left(J:_{S} f^{-1}\left(\bar{J}^{*}\right)\right)=f^{-1}\left(\tau_{R}\right)$, we are done. To see this we note that

$$
v \in \bigcap_{I \subseteq J}\left(J:_{S} f^{-1}\left(\bar{J}^{*}\right)\right)
$$

if and only if

$$
v \in\left(J:_{S} f^{-1}\left(\bar{J}^{*}\right)\right) \text { for all } \bar{J} \subseteq R
$$

if and only if

$$
v f^{-1}\left(\bar{J}^{*}\right) \subseteq J \text { for all } \bar{J} \subseteq R
$$

if and only if

$$
f(v) \bar{J}^{*} \subseteq \bar{J} \text { for all } \bar{J} \subseteq R
$$

if and only if

$$
f(v) \in\left(\bar{J}:_{R} \bar{J}^{*}\right) \text { for all } \bar{J} \subseteq R
$$

if and only if

$$
f(v) \in \tau_{R}
$$

if and only if

$$
v \in f^{-1}\left(\tau_{R}\right)
$$

Thus $w \in\left(f^{-1}\left(\tau_{R}\right)^{[p]}: f^{-1}\left(\tau_{R}\right)\right)$.

Notice that the proof holds for any $q=p^{e}$. Fedder has noted that to discuss $F$-purity of a ring $R$ we must assume that $R$ is reduced. The proof of the next theorem relies on the fact that in an $F$-finite, $F$-pure ring, that the test ideal is nonzero and has positive height. To see that the test ideal is nonzero, recall the following theorem of Hochster and Huneke: 
Theorem 3.2. [HH2, Theorem 3.4] Let $R$ be an F-finite reduced ring of characteristic $p$. Let $c$ be any nonzero element of $R$ such that $R_{c}$ is regular. Then $c$ has a power which is a test element.

Theorem 3.3. Let $R=S / I$ be an $F$-finite, F-pure ring. Let $\tau_{R}$ be the pullback of the test ideal of $R$ in $S$. Then $S / \tau_{R}$ is F-pure and ht $(\tau) \geq 1$.

Proof. Recall that $F$-pure rings are reduced. For all minimal primes $P / I$ in $R$ we know that $R_{P / I}=(S / I)_{P / I}=S_{P} / P$, which is a field. The regular locus is open in an excellent Noetherian local ring. A theorem of Kunz [Ku2] shows that $F$-finite rings are excellent. We can conclude that the regular locus of $R$ is nonempty. Thus if we choose $c$ to be an element such that the primes not containing $c$ are contained inside the regular locus, then $R_{c}$ is regular, and thus some power is in the test ideal by Theorem 3.2. Since $R$ is reduced $c$ is not nilpotent, so it follows that $h t((c))=1$. Therefore, $\operatorname{ht}\left(\tau_{R}\right) \geq 1$.

Define $R_{1}=R / \tau_{R} \cong S / f^{-1}\left(\tau_{R}\right)$. Since $R$ is $F$-pure, Fedder's $F$-purity criterion, Theorem 2.5, gives that $\left(I^{[p]}: I\right) \nsubseteq \mathfrak{m}^{[p]}$. Theorem 3.1 implies that

$$
\left(f^{-1}\left(\tau_{R}\right)^{[p]}: f^{-1}\left(\tau_{R}\right)\right) \nsubseteq \mathfrak{m}^{[p]} .
$$

Thus another application of Theorem 2.5 implies that $R_{1}$ is $F$-pure.

An immediate corollary of Theorem 3.3 is the following filtration of $R$ by test ideals. In [S2] Smith shows that the test ideal is a $D$-module, where $D$ is the ring of differential operators on $R$; thus, the following filtration is also an example of a filtration of $R$ by $D$-modules.

Corollary 3.4. Let $R=S / I$ be an F-finite, F-pure ring. Then there exists a unique filtration $\tau_{0} \subseteq \tau_{1} \subseteq \ldots \subseteq \tau_{c-1} \subseteq \tau_{c}$ of $R$ such that $\tau_{i+1} / \tau_{i}$ is the test ideal for $R / \tau_{i}$ and the $R / \tau_{i}$ are all $F$-pure.

Proof. Applying Theorem 3.3 to $R_{i}=S / \tau_{i-1}$ where $\tau_{i-1}$ is the pullback of the test ideal of $R_{i-1}$ in $S$, we see that $R_{i}$ is $F$-pure for all $i$ and we get the unique filtration $0 \subseteq \tau_{0} \subseteq \tau_{1} / I \ldots \subseteq \tau_{i} / I \subseteq \ldots \tau_{c}$ where is the last such $i$ such that $\tau_{c} \neq R$ and in which all of the $R_{i}$ are $F$-pure and ht $\left(\tau_{R_{i+1}} / \tau_{R_{i}}\right) \geq 1$.

In fact, Theorem 3.3 implies the following theorem of Fedder and Watanabe.

Theorem 3.5 ([FW, Proposition 2.5]). If $R$ is an F-pure ring, then the test ideal is radical.

Goto and Watanabe have classified one-dimensional $F$-pure rings containing an algebraically closed field in [GW]. They prove the following:

Theorem 3.6. [GW, Theorem 1.1] Let $(R, \mathfrak{m}, k)$ be a one-dimensional local ring with $k$ algebraically closed and of positive characteristic. If $R$ is $F$-finite, then $R$ is F-pure if and only if $\hat{R}$ is isomorphic to $k\left[\left[x_{1}, \ldots, x_{r}\right]\right] /\left(x_{i} x_{j}\right)_{i<j}$, where $r$ is the number of associated primes of $\hat{R}$.

This theorem tempts us to find an algorithm to compute the test ideal of reduced rings $R$ where $R=T / I$ and $I$ is an ideal generated by monomials $x_{1}, \ldots, x_{n}$ in a $T$-sequence where $x_{i}=f\left(X_{i}\right)$ where $f$ maps $A\left[X_{1}, \ldots, X_{n}\right]$ to $T$. For a thorough discussion on monomials see [Ei, 15.1], and for a reference to monomials in the tight closure context refer to [HH1, 7.3]. For the following theorem we note that the sum of two ideals generated by monomials is an ideal generated by monomials, and the intersection of two such ideals is also generated by monomials. 
Theorem 3.7. Let $R=T / I$ with $T$ as above, where $I=P_{1} \cap \ldots \cap P_{n}$ is generated by square-free monomials and $R / P_{i}$ are regular. Set $J=\Sigma\left(P_{1} \cap \ldots \cap \hat{P}_{i} \cap \ldots \cap P_{n}\right)$. Then $J=\tau=\tau_{\text {par }}$.

Proof. To show that $J \subseteq \tau$ we need only see that $\left(P_{1} \cap \ldots \cap \hat{P}_{i} \cap \ldots \cap P_{n}\right) I^{*} \subseteq I$. However, since $I^{*}=\bigcap\left(\bar{I}+P_{i}\right)$,

$$
\begin{gathered}
\left(P_{1} \cap \ldots \cap \hat{P}_{i} \cap \ldots \cap P_{n}\right) I^{*} \subseteq\left(P_{1} \cap \ldots \cap \hat{P}_{i} \cap \ldots \cap P_{n}\right)\left(I+P_{i}\right) \\
=\left(P_{1} \cap \ldots \cap \hat{P}_{i} \cap \ldots \cap P_{n}\right) I \subseteq I .
\end{gathered}
$$

To show the other inclusion, take a nonzero $x_{i} \in\left(P_{1} \cap \ldots \cap \hat{P}_{i} \cap \ldots \cap P_{n}\right)$. Then $x_{1}+\ldots+x_{n}$ is not in the union of minimal primes and it is the generator of a parameter ideal. Note that $x_{i} \in\left(x_{1}+\ldots+x_{n}\right)^{*}$, since $\left(x_{1}+\ldots+x_{n}\right) x_{i}^{q}=$ $\left(x_{1}+\ldots+x_{n}\right)^{q} x_{i}$ for all $i$. Take a minimal prime $\mathfrak{p}$ over $J$. If $\tau \nsubseteq J$, then by a prime avoidance argument there exists a $c \in \tau$ such that $c \notin \mathfrak{p}$. Thus $c$ is a unit in $R_{\mathfrak{p}}$ and $\left(x_{1}+\ldots+x_{n}\right)^{*} R_{\mathfrak{p}}=\left(x_{1}+\ldots+x_{n}\right) R_{\mathfrak{p}}$. In $R_{\mathfrak{p}}, x_{i} \in\left(x_{1}+\ldots+x_{n}\right) R_{\mathfrak{p}}$, i.e. $x_{i}=a\left(x_{1}+\ldots+x_{n}\right)$. We see that $(1-a) x_{i}=a\left(x_{1}+\ldots+\hat{x}_{i}+\ldots+x_{n}\right)$. Since $R_{\mathfrak{p}}$ is local, either $a$ or $1-a$ is a unit. Thus either $x_{i} \in\left(x_{1}+\ldots+\hat{x}_{i}+\ldots+x_{n}\right)$, which implies $x_{i} \in P_{i}$ or $\left(x_{1}+\ldots+\hat{x}_{i}+\ldots+x_{n}\right) \in\left(x_{i}\right)$, which implies

$$
\left(x_{1}+\ldots+\hat{x}_{i}+\ldots+x_{n}\right) \in\left(P_{1} \cap \ldots \cap \hat{P}_{i} \cap \ldots \cap P_{n}\right) .
$$

From both of these conclusions we can conclude $x_{i}=0$ for some $i$. But we assumed the $x_{i}$ were nonzero.

Note that this argument also shows that $J=\tau_{\text {par }}$, since we can assume that $I$ in the first sentence of the proof is a parameter ideal and replace $\tau$ by $\tau_{\text {par }}$ throughout the rest of the proof.

Recall the strong test ideal:

Definition 3.8. A strong test ideal is an ideal $J \subseteq \tau$ such that $J I^{*}=J I$. We denote by $J=\tau_{\text {str }}$ the largest one.

Corollary 3.9. Under all the assumptions of Theorem 3.7, $J=\tau_{\text {str }}$.

Proof. We know that $\tau_{s t r} \subseteq \tau=J$. And

$$
\begin{gathered}
\left(P_{1} \cap \ldots \cap \hat{P}_{i} \cap \ldots \cap P_{n}\right) I^{*} \subseteq\left(P_{1} \cap \ldots \cap \hat{P}_{i} \cap \ldots \cap P_{n}\right)\left(I+P_{i}\right) \\
=\left(P_{1} \cap \ldots \cap \hat{P}_{i} \cap \ldots \cap P_{n}\right) I
\end{gathered}
$$

implies that $J I^{*}=J I$. Thus $\tau_{s t r}=J$.

Note that we can also prove Theorem 3.7 as a corollary of the following more general theorem.

Theorem 3.10. Let $R$ be an F-pure ring and $I$ and $J$ be ideals in $R$ such that $I \cap J=(0)$. Suppose also that $\operatorname{Ann}(I)=J$ and $\operatorname{Ann}(J)=I$. Let $\tau_{R / I}$ and $\tau_{R / J}$ be test ideals in $R / I$ and $R / J$ lifted back to $R$ respectively. If $\mathfrak{a}=I \cap \tau_{R / J}+J \cap \tau_{R / I}$ is self-radical, then $\tau=\mathfrak{a}$.

Proof. We need only see that $\mathfrak{a} \subseteq \tau$, since the other containment follows from a proof similar to showing that $\tau \subseteq J$ in Theorem 3.7. It is enough to show that $I \tau_{R / J} \subseteq \tau$ and $J \tau_{R / I} \subseteq \tau$, since $I \tau_{R / J}+J \tau_{R / I} \subseteq I \cap \tau_{R / J}+J \cap \tau_{R / I} \subseteq$ $\operatorname{Rad}\left(I \cap \tau_{R / J}\right)+\operatorname{Rad}\left(J \cap \tau_{R / I}\right) \subseteq \operatorname{Rad}\left(I \tau_{R / J}\right)+\operatorname{Rad}\left(J \tau_{R / I}\right) \subseteq \operatorname{Rad}\left(I \tau_{R / J}+J \tau_{R / I}\right) \subseteq$ 
a. If $R$ is $F$-pure then $[\mathrm{FW}]$ shows $\tau$ is radical. Let $\mathfrak{b}$ be an ideal in $R, \mathfrak{b}^{*}=$ $(\mathfrak{b}+I)^{*} \cap(\mathfrak{b}+J)^{*}$, where $(\mathfrak{b}+I)^{*}$ is the pullback of $(\mathfrak{b}+I)^{*}$ in $R / I$ and $(\mathfrak{b}+J)^{*}$ is similarly defined in $R / J$. Note that $\left(I \tau_{R / J}\right) \mathfrak{b}^{*} \subseteq I(\mathfrak{b}+J) \subseteq I \mathfrak{b} \subseteq \mathfrak{b}$. By symmetry $J \tau_{R / I} \mathfrak{b}^{*} \subseteq \mathfrak{b}$.

To give some examples of $F$-pure filtrations we use the above theorems to prove the following:

Theorem 3.11. Let $T=k\left[\left[x_{1}, \ldots, x_{n}\right]\right]$ or $T=k\left[x_{1}, \ldots, x_{n}\right]$. Set $R=T / I$, where

$$
I=\left\langle x_{i_{1}} \ldots x_{i_{d}}\right\rangle_{1 \leq i_{1}<\ldots<i_{d} \leq n} .
$$

Then $\tau=\left\langle x_{i_{1}} \ldots \hat{{i_{r}}_{r}} \ldots x_{i_{d}}\right\rangle_{1 \leq i_{1}<\ldots<i_{d} \leq n}$.

Proof. Note that $I$ is generated by $\left(\begin{array}{l}n \\ d\end{array}\right)$ elements each missing $(n-d) x_{i}$ 's. If we choose $(n-d+1)$ distinct $x_{i}$ 's $\left\{x_{j_{1}}, \ldots, x_{j_{n-d+1}}\right\}$, then for a fixed $\left\{x_{i_{1}}, \ldots, x_{i_{d}}\right\}$ some $x_{j_{n}}$ is a factor of $x_{i_{1}} \ldots x_{i_{d}}$ (i.e. $j_{n}=i_{m}$, where $x_{i_{m}} \in\left\{x_{i_{1}}, \ldots, x_{i_{d}}\right\}$ ), since $x_{i_{1}} \ldots x_{i_{d}}$ is only missing $(n-d) x_{i}$ 's. Take $P_{j_{1} \ldots j_{n-d+1}}=\left(x_{j_{1}}, \ldots, x_{j_{n-d+1}}\right)$. The $P_{j_{1} \ldots j_{n-d+1}}$ are minimal primes and there are $\left(\begin{array}{c}n \\ n-d+1\end{array}\right)$ of them. Theorem 2.9 says that $\tau=\Sigma \bigcap_{j_{i} \neq k_{i}} P_{j_{1} \ldots j_{n-d+1}}$ for some $\left\{k_{1}, \ldots, k_{n-d+1}\right\}$. But

$$
\bigcap_{j_{i} \neq k_{i}} P_{j_{1} \ldots j_{n-d+1}}=\left(x_{l_{1}} \ldots x_{l_{d-1}},\left\langle x_{k_{r_{1}}} x_{k_{r_{2}}} \ldots x_{k_{r_{d}}}\right\rangle\right)
$$

if $n-d+1 \geq d$, or

$$
\bigcap_{j_{i} \neq k_{i}} P_{j_{1} \ldots j_{n-d+1}}=\left(x_{l_{1}} \ldots x_{l_{d-1}},\left\langle x_{k_{1}} \ldots x_{k_{n-d+1}} x_{l_{s_{1}}} \ldots x_{l_{s_{2 d-n-1}}}\right\rangle\right),
$$

where $\left\{l_{1}, \ldots l_{d-1}\right\}$ is the complement of $\left\{k_{1}, \ldots, k_{n-d+1}\right\}$ in $\{1, \ldots, n\}$. Thus

$$
\tau=\left\langle x_{l_{1}} \ldots x_{l_{d-1}}\right\rangle_{1 \leq l_{1}<\ldots<l_{d-1} \leq n}=\left\langle x_{i_{1}} \ldots{\hat{i_{r}}} \ldots x_{i_{d}}\right\rangle_{1 \leq i_{1}<\ldots<i_{d} \leq n} .
$$

Example 3.12. 1. Let $R=k[[x, y, z]] /(x y, x z, y z)$, where $k$ is algebraically closed. By Theorem 3.6, $R$ is $F$-pure. By Theorem 3.3, then $R / \tau$ is also $F$-pure. Thus we need to compute $\tau$. By Theorem $3.11 \tau=(x, y, z)=\mathfrak{m}$. So the filtration is $(x y, x z, y z) \subseteq \mathfrak{m} \subseteq R$.

2. Let $R=k[[x, y, z, w]] /(x y z, x y w, x z w, y z w)$, where $k$ is algebraically closed. By Fedder's $F$-purity criterion, $R$ is $F$-pure. By 3.3 we also know that $R / \tau=R_{1}$ is $F$-pure. By Theorem 3.11, $\tau=(x y, x z, x w, y z, y w, z w)$. As in the previous example, $\tau_{R_{1}}=\mathfrak{m}$. Thus the $F$-pure filtration is

$$
(x y z, x y w, x z w, y z w) \subseteq(x y, x z, x w, y z, y w, z w) \subseteq \mathfrak{m} \subseteq k[[x, y, z, w]] .
$$

3. Let $R=k[[x, y, z]] /(x y, y z)$, where $k$ is algebraically closed. By Fedder's $F$-purity criterion, $R$ is $F$-pure. Note that $P_{1}=(y)$ and $P_{2}=(x, z)$. By Theorem 3.7, $\tau=(x, y, z)$. So the filtration is $(x y, x z, y z) \subseteq \mathfrak{m} \subseteq R$.

4. Let $R=k[[x, y, z, w]] /(x y, z w)$, where $k$ is algebraically closed. Using Fedder's $F$-purity criterion, $R$ is $F$-pure. By 3.3 we also know that $R / \tau=R_{1}$ is $F$-pure. By Theorem 3.11, $\tau=(x y, x z, x w, y z, y w, z w)$. As in the previous example, $\tau_{R_{1}}=\mathfrak{m}$. Thus the $F$-pure filtration is

$$
(x y, z w) \subseteq(x y, x z, x w, y z, y w, z w) \subseteq \mathfrak{m} \subseteq k[[x, y, z, w]] .
$$

One might wonder if it is possible to classify $n$-dimensional $F$-pure rings containing an algebraically closed field up to isomorphism using the above filtration. 


\section{Powers of tight ClOsures of IDEALS}

What power of $I^{*}$ is contained in $I$ ? In an $F$-pure ring we have the following theorem of Huneke [Hu, Proposition 4.9]:

Theorem 4.1. Let $R$ be a d-dimensional Noetherian Cohen Macaulay local ring which is F-pure. Then for all ideals I

$$
\overline{I^{d+1}} \subseteq I .
$$

Theorem 4.1 can be improved slightly. The proof is similar to the proof of 4.1.

Theorem 4.2. Let $R$ be a d-dimensional Noetherian Cohen Macaulay local ring which is F-pure. Then for all ideals $I$

$$
\overline{I^{d+n}} \subseteq I^{n} .
$$

Proof. Without loss of generality assume that $I$ is a minimal reduction. Thus $I$ is generated by at most $d$ elements. Take $u \in \overline{I^{d+n}}$. Fix an integer $k$ such that $u^{k+m} \in I^{m(d+n)}$. Set $m=q-k$. Then $u^{q} \in I^{(q-k)(d+n)}$. Rewriting the power $(q-k)(d+n)=q(d+n-1)+q-k(d+n)$ and choosing $q>k(d+n)$, we see that $u^{q} \in I^{q(d+n-1)} \subseteq I^{[q]} I^{q(d+n-2)} \subseteq \ldots \subseteq\left(I^{[q]}\right)^{n-1} I^{q d} \subseteq\left(I^{[q]}\right)^{n}=\left(I^{n}\right)^{[q]}$. Since $R$ is $F$-pure, then $u \in I^{n}$.

Both of the above theorems imply $\left(I^{*}\right)^{d+1} \subseteq I$. But we would like a better bound. Corollary 3.4 gives us a hint on how to find this bound in $F$-pure rings. Recall the setup for Corollary 3.4. Let $R=S / I$ be an $F$-finite, $F$-pure ring. As noted earlier in $F$-pure local rings test elements exist. Thus define $\tau_{0}$, the test ideal of $R$. As we noted before, $\operatorname{ht}\left(\tau_{0}\right) \geq 1 . R / \tau_{0}$ is again $F$-pure, so again we can define $\tau_{1}$, the test ideal of $R / \tau_{0}$. Continue until $\tau_{c}=R$ for some $c$. Now our filtration looks like $\tau_{0} \subseteq \tau_{1} \subseteq \ldots \subseteq \tau_{c-1} \subseteq \tau_{c}$ such that $\tau_{i+1} / \tau_{i}$ is the test ideal for $R / \tau_{i}$ and the $R / \tau_{i}$ are all $F$-pure and ht $\left(\tau_{i+1} / \tau_{i}\right) \geq 1$. To make the following theorems' proofs and statements easier we shall relabel the above filtration, setting $i=c-i$. Thus our new filtration looks like $\tau_{c} \subseteq \tau_{c-1} \subseteq \ldots \subseteq \tau_{1} \subseteq \tau_{0}$, with $\tau_{i} / \tau_{i+1}$ begin the test ideal for $R / \tau_{i+1}$ and ht $\left(\tau_{i} / \tau_{i+1}\right) \geq 1$. To make the proofs of our theorems more understandable, recall

Theorem 4.3. [HH3, Theorem 6.24] Let $\phi: R \rightarrow S$ be a homomorphism of $F$ finite reduced Noetherian rings of characteristic $p$. Let $I$ be an ideal of $R$ and $w \in I^{*}$. Then $\phi(w)$ is in the tight closure of IS.

Theorem 4.4. Let $R=S / I, S$ an F-finite regular local ring. Suppose that $R$ is $F$ pure and $J$ is an ideal of $R$. Let $c+1$ be the length of the unique filtration described above. For some $t \in\{0,1, \ldots, c\}$, we have $J \subseteq \tau_{i}$ for $i \leq t$. Then $\left(J^{*}\right)^{c-t+2} \subseteq J$.

Proof. Note that $\operatorname{Rad}(J)=\operatorname{Rad}\left(J^{*}\right)$. Thus if $J \subseteq \tau_{t}$ then $J^{*} \subseteq \tau_{t}$, since $\tau_{t}$ is reduced. We know by Theorem 4.3 that if $x$ is in the tight closure of an ideal $J$ of $R$, then $x+\tau_{i}$ is in the tight closure of $J+\tau_{i}$ in $R / \tau_{i}$ for all $i$. In the $\operatorname{ring} R / \tau_{t+1}$ we know the test ideal is $\tau_{t} / \tau_{t+1}$. Thus by persistence we know that

$$
\tau_{t} J^{*} \subseteq J+\left(\tau_{t+1} \cap J^{*}\right) .
$$

Note that multiplying by $J^{*}$ gives us the following:

$$
\tau_{t}\left(J^{*}\right)^{2} \subseteq J+\left(\tau_{t+1} \cap J^{*}\right) J^{*} \subseteq J+\left(\tau_{t+2} \cap J^{*}\right),
$$


again by persistence. If we continue to multiply by $J^{*}$, after $c-t+1$ steps we have

$$
\tau_{t}\left(J^{*}\right)^{c-t+1} \subseteq J
$$

Thus $\left(J^{*}\right)^{c-t+2} \subseteq J$.

This result can be slightly improved if $\tau_{t}$ is not contained in the union of associated primes of $J$.

Theorem 4.5. Let $R=S / I, S$ an $F$-finite regular local ring. Suppose that $R$ is $F$-pure and $J / I$ is an ideal of $R$. Let $c+1$ be the length of the unique filtration described above. For some $t \in\{0,1, \ldots, c\}$, we have $J \subseteq \tau_{i}$ for $i \leq t$, and if $\tau_{t} \not \subseteq \operatorname{Ass}(J)$ then $\left(J^{*}\right)^{c-t+1} \subseteq J$.

Proof. Following the proof of Theorem 4.4, we see that

$$
\tau_{t}\left(J^{*}\right)^{c-t+1} \subseteq J
$$

Thus

$$
\left(J^{*}\right)^{c-t+1} \subseteq\left(J: \tau_{t}\right)=J
$$

since $\tau_{t} \nsubseteq \operatorname{Ass}(J)$.

We see from 4.5 that the $\tau_{i}$ not contained in the associated primes of an ideal $J / I$ can virtually be ignored. In effect, for each $J / I$ we can reduce the length of the filtration by successive test ideals from $c+1$ to $c-s$, where $s$ is the largest integer such that $\tau_{s} \nsubseteq \operatorname{Ass}(J)$. We relabel $\tau_{s}$ by $\tau_{0}$, and for all $i>s, \tau_{i}$ becomes $\tau_{i-s}$. Thus our new filtration looks like $\tau_{c-s-1} \subseteq \ldots \subseteq \tau_{1} \subseteq \tau_{0}$, and it has all the same properties as the above filtration. We can reformulate the statements of both 4.4 and 4.5 in terms of our new filtration in the following two theorems respectively:

Theorem 4.6. Let $R=S / I, S$ an $F$-finite regular local ring. Suppose that $R$ is $F$-pure and $J / I$ is an ideal of $R$. Let $c-s$ be the length of the unique filtration described above. For some $r \in\{0,1, \ldots, c-s-1\}$ let $J \subseteq \tau_{i}$ for $i \leq r$. Then $\left(J^{*}\right)^{c-s-r+1} \subseteq J$.

Theorem 4.7. Let $R=S / I, S$ an $F$-finite regular local ring. Suppose that $R$ is $F$-pure and $J / I$ is an ideal of $R$. Let $c-s$ be the length of the unique filtration described above. If $J \subseteq \tau_{0}$ then $\left(J^{*}\right)^{c-s} \subseteq J$.

From these new statements we can estimate bounds in terms of heights of the associated primes of the ideal $J$. Note that since $\tau_{0}$ is the only element of our filtration not contained in any associated primes of $J$ and $\operatorname{ht}\left(\tau_{i}\right)>\operatorname{ht}\left(\tau_{i+1}\right)$ for all $i$ (since $\operatorname{ht}\left(\tau_{i} / \tau_{i+1}\right) \geq 1$ ) it follows that $c-s-1 \leq \max \left\{\operatorname{ht}\left(P_{i}\right) \mid P_{i} \subseteq \operatorname{Ass}(J)\right\}$. As a corollary of 4.6 and 4.7 we have

Corollary 4.8. Let $R=S / I, S$ an F-finite regular local ring. Suppose that $R$ is $F$-pure and $J / I$ is an ideal of $R$. Let $c-s$ be the length of the unique filtration described above. For some $r \in\{0,1, \ldots, c-s-1\}$ let $J \subseteq \tau_{i}$ for $i \leq r$. Then $\left(J^{*}\right)^{h-r+2} \subseteq J$, where $h=\max \left\{\operatorname{ht}\left(P_{i}\right) \mid P_{i} \subseteq \operatorname{Ass}(J)\right\}$.

Corollary 4.9. Let $R=S / I, S$ an $F$-finite regular local ring. Suppose that $R$ is $F$-pure and $J / I$ is an ideal of $R$. Let $c-s$ be the length of the unique filtration described above. If $J \subseteq \tau_{0}$ then $\left(J^{*}\right)^{h+1} \subseteq J$, where $h=\max \left\{\operatorname{ht}\left(P_{i}\right) \mid P_{i} \subseteq \operatorname{Ass}(J)\right\}$. 


\section{REFERENCES}

[BH] Bruns, W. and Herzog, J., Cohen-Macaulay Rings, Cambridge University Press, Cambridge, 1993. MR 95h:13020

[Ei] Eisenbud, D., Commutative Algebra: With a View Toward Algebraic Geometry, Springer-Verlag, New York, 1995. MR 97a:13001

[Fe] Fedder, R., F-purity and rational singularity, Trans. Amer. Math. Soc 278 (1983), 461-480. MR 84h:13031

[FW] Fedder, R. and Watanabe, K., A characterization of F-regularity in terms of F-purity, Commutative Algebra, MSRI Publications, Springer-Verlag, 1989, pp. 227-245. MR 91k:13009

[Gl] Glassbrenner, D, Strong F-regularity in images of regular rings, Proc. Amer. Math. Soc. 124 (1996), 345-353. MR 96d:13004

[GW] Goto, S, Watanabe, K, The structure of one-dimensional F-pure rings, J. of Alg. 49 (1977), 415-421. MR 56:11989

[Ha] Hara, N., A characterization of rational singularities in terms of injectivity of Frobenius maps, preprint.

[Ho] Hochster, M., Cyclic purity versus purity in excellent Noetherian rings, Trans. Amer. Math. Soc. 231 (1977), 463-488. MR 57:3111

[HH1] Hochster, M. and Huneke, C., Tight closure, invariant theory, and the Briançon-Skoda theorem, J. Amer. Math. Soc. 3 (1990), 31-116. MR 91g:13010

[HH2] Hochster, M. and Huneke, C., Tight closure and strong F-regularity, Mémoires de la Société Mathématique de France 38 (1989), 119-133. MR 91i:13025

[HH3] Hochster, M. and Huneke, C., F-regularity, test elements and smooth base change, Trans. Amer. Math. Soc. 346 (1994), 1-62. MR 95d:13007

[Hu] Huneke, C., Hilbert functions and symbolic powers, Mich. Math. J. 34 (1987), 293-318. MR 89b: 13037

[HS] Huneke, C. and Smith, K., Tight closure and the Kodaira vanishing theorem, J. Reine Angew. Matth. 484 (1997), 127-152. CMP 97:09

[Ku1] Kunz, E., Characterization of regular local rings for characteristic p, Amer. J. Math 91 (1969), 772-784. MR 40:5609

[Ku2] Kunz, E., On Noetherian rings of characteristic p, Am. J. Math. 98 (1976), 999-1013. MR 55:5612

[S1] Smith, K., Test ideals in local rings, Trans. Amer. Math. Soc. 347 (1995), 3453-3472. MR 96c: 13008

[S2] Smith, K., The D-module structure of F-split rings, Math Research Letters 2 (1995), 377-386. MR 96j:13024

Department of Mathematics, University of California, Los Angeles, California 90024 Current address: Department of Mathematical Sciences, Virginia Commonwealth University, Richmond, Virginia 23284

E-mail address: jcvassil@saturn.vcu.edu 